\title{
130 170 GHz 平坦毫米波噪声信号产生技术
}

\author{
孙粤辉 ${ }^{1}$ ，郭亚 ${ }^{2} ，$ 王云才 ${ }^{1 *}$ ，刘文杰 ${ }^{1}$ ， 黄海碧 ${ }^{1}$ ， 黄奕敏 ${ }^{1} ，$ 秦玉文 ${ }^{1}$
}

1. 广东工业大学信息工程学院, 广东省信息光子技术重点实验室, 广州 510006

2. 西北工业大学电子信息学院, 西安 710072

* 通信作者. E-mail: wangyc@gdut.edu.cn

收稿日期: 2021-12-25; 修回日期: 2022-01-28; 接受日期: 2022-02-14; 网络出版日期: 2022-11-10

国家自然科学基金 (批准号: 61927811，61961136002，61731014，62150410435) 和“珠江人才计划” 引进创新创业团队项目基金 资助

摘要 本文提出了一种基于三束非相干光混频产生 D 波段平坦毫米波噪声信号的方法. 通过设置与 调节三束光的中心波长与滤波光谱宽度, 利用高速光电探测器同时实现光生毫米波噪声信号的频率范 围与功率谱平坦度有效可控. 理论分析了三束高斯型非相干光的中心波长差、滤波光谱宽度与预设频段 噪声功率谱平坦度的关系. 实验中, 以单行载流子光电探测器 (UTC-PD) 为光混频器, 在 130 170 GHz 产生了超噪比 $(\mathrm{ENR})>35 \mathrm{~dB}$ 、平坦度低至 $\pm 2.5 \mathrm{~dB}$ 的 D 波段毫米波噪声信号.

关键词噪声发生器, 毫米波噪声, 光子混频, 超噪比, 平坦度

\section{1 引言}

频率范围为 110 170 GHz 的 D 波段毫米波, 具有频带宽、大气传播窗口衰减小的优势, 满足超高 速大容量的数据传输需求, 是未来 $\mathrm{B} 5 \mathrm{G} / 6 \mathrm{G}$ 无线移动通信的候选频段 ${ }^{[1]}$, 也在雷达、成像、安检、医 学诊断等领域有良好的应用空间 ${ }^{[2 \sim 5]}$. D 波段毫米波的各种系统应用离不开高频器件与设备的关键 支撑 ${ }^{[6]}$. 作为高频器件必不可少的测试仪器, 毫米波噪声发生器通过向待测器件中输入确定的噪声信 号就能够表征待测期间的性能, 如: 测试接收机灵敏度 ${ }^{[7]}$ 、分析探测器的频率响应 ${ }^{[8]}$ 、测量放大器噪

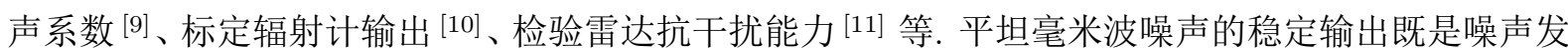
生器性能优异的标识, 也在提升 D 波段器件、模块或系统性能等方面扮演着不可或缺的角色.

实现高频毫米波噪声发生器的技术方案主要有两种. 一种是基于电子学的方法, 核心是控制放大

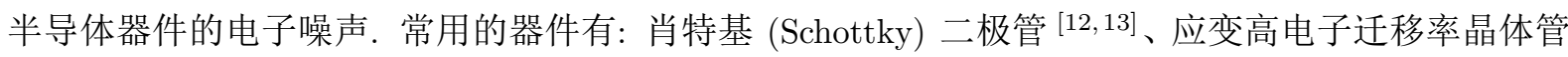
(metamorphic high-electron-mobility transistor, MHEMT) ${ }^{[14,15]}$ 、异质结晶体管 (heterojunction bipolar transistor, HBT) ${ }^{[16]}$ 等. 利用该方法产生的噪声频率受限于器件自身的 “电子瓶颈”, 高频处功率下降

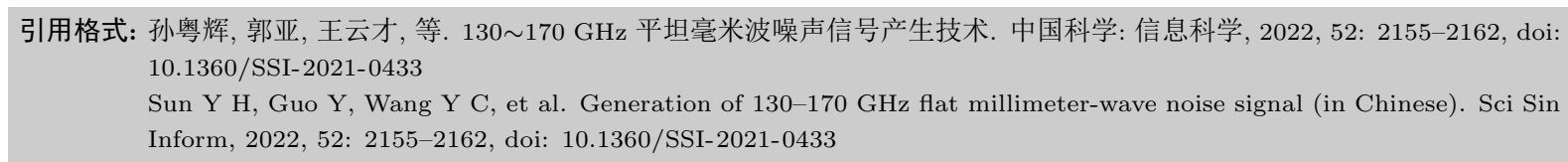


明显, 超噪比 (excess noise ratio, ENR) 一般不超过 $20 \mathrm{~dB}$. 通过该方法很难得到 D 波段连续平坦的 噪声. 另外一种是基于光子学的方法, 主要利用非相干光源的光谱 - 频谱映射关系 ${ }^{[17]}$ 并借助高速光 电探测器产生毫米波噪声, 如掺饵光纤放大器 (erbium-doped fiber amplifier, EDFA)、半导体光放大器 (semiconductor optical amplifier, SOA)、超辐射发光二极管 (super luminescent diode, SLD) 等. 非相干 光源产生的放大自发辐射 (amplified spontaneous emission, ASE) 噪声的光谱线宽可达 $30 \sim 40 \mathrm{~nm}$, 对 应频谱覆盖范围可达 3 5 THz.

相较于电子学的方法, 光子学方法具有可产生 D 波段乃至更高频的宽带平坦噪声的优势, 因此, 近年来该方面的研究备受关注. 2009 年, 日本 Nagatsuma 等 ${ }^{[18]}$ 在毫米波成像系统中将 ASE 光源直接 耦合进单行载流子光电探测器 (uni-travelling-carrier photodetector, UTC-PD), 产生了 90 140 GHz 频 段毫米波噪声. 2020 年, Vidal ${ }^{[19]}$ 将一束线宽 $1 \mathrm{~nm}$ 的 ASE 光经 EDFA 放大后耦合进带宽为 $20 \mathrm{GHz}$ 的光电探测器得到微波噪声. 直接对非相干光源光电转换得到的电噪声功率较低, 无法满足功率需 求较高的设备检测与标定. 2008 年, Song 等 ${ }^{[20]}$ 提出利用阵列波导光栅 (arrayed waveguide grating, AWG) 对宽带 ASE 光源滤波得到多组不同波长的两个噪声光谱, 经过耦合放大后通过高速 UTC-PD 转换为 $295 \sim 395 \mathrm{GHz}$ 的电噪声. 与直接对非相干光源探测相比, 此方案可将噪声功率提升 $15 \sim 17 \mathrm{~dB}$. 不足之处在于, 人工切换 AWG 滤波通道导致无法得到连续平坦的噪声. 在测量待测器件噪声系数时, 不平坦的毫米波噪声将导致噪声系数的动态精度降低 ${ }^{[9]} .2021$ 年, Sun 等 ${ }^{[21]}$ 利用多个不同波长 ASE 光混频, 实验产生了 $50 \mathrm{GHz}$ 平坦的毫米波噪声信号.

本文提出一种将非相干光源整形滤波与高速 UTC-PD 相结合混频产生 D 波段平坦毫米波噪声 信号的新方法. 首先对宽带 ASE 光源整形滤波控制输出的三束高斯 (Gaussian) 型 ASE 光的中心波 长与滤波光谱宽度, 再将三束光耦合放大后混频产生指定频段的平坦毫米波噪声信号. 通过数值模拟 计算与实验, 对不同滤波光谱宽度的三光混频得到的 D 波段毫米波噪声功率谱平坦度进行对比分析. 在实验中, 依据 UTC-PD 提供的最大工作带宽, 在 130 170 GHz 产生了超噪比 $>35 \mathrm{~dB}$ 、平坦度低至 $\pm 2.5 \mathrm{~dB}$ 的 D 波段毫米波噪声信号. 本方法借助可编程光滤波器对宽带 ASE 光源进行滤波选择, 使 得毫米波噪声信号的产生频段与平坦度灵活可控, 对未来更高频、更大带宽、更平坦的毫米波噪声信 号的产生具有重要参考价值.

\section{2 基本原理}

图 1 为不同中心波长的三束高斯型 ASE 光混频产生特定频段毫米波噪声信号的原理性示意图. 首先, 宽谱的 ASE 光源经整形滤波后输出三束中心波长分别为 $\lambda_{1}, \lambda_{2}, \lambda_{3}$ 的高斯型 ASE 光. 毫米波 噪声信号频段的下限频率 $f_{\mathrm{L}}$ 由 $\left(\mathrm{c} / \lambda_{1}-\mathrm{c} / \lambda_{2}\right)$ 决定, 而上限频率 $f_{\mathrm{H}}$ 取决于 $\left(\mathrm{c} / \lambda_{1}-\mathrm{c} / \lambda_{3}\right)$, 其中, $\mathrm{c}$ 为 光速. 通过控制滤波的带宽, 将三束光耦合放大后在高速光电探测器中混频, 产生 $f_{\mathrm{L}} \sim f_{\mathrm{H}}$ 频率范围 的平坦毫米波噪声信号.

三束滤波光谱宽度相同的高斯型 $\mathrm{ASE}$ 光耦合后的光谱密度 $S_{\mathrm{ASE}}(\nu)$ 可表示为 ${ }^{[21]}$

$$
S_{\mathrm{ASE}}(v)=\frac{S_{\mathrm{ASE}, 0}}{3} \sum_{k=1}^{3} \exp \left(-\frac{\left(v-v_{k}\right)^{2}}{2 \sigma^{2}}\right),
$$

其中, $\nu$ 为光谱频率 $(\nu=\mathrm{c} / \lambda) ; S_{\mathrm{ASE}, 0}$ 为光谱中心频率的谱密度峰值幅度; $\sigma$ 为高斯分布的标准差, 与 滤波光谱宽度 (full-width at half-maximum, FWHM) $\Lambda$ 的关系为

$$
\Lambda=2 \sqrt{\ln 4} \sigma .
$$



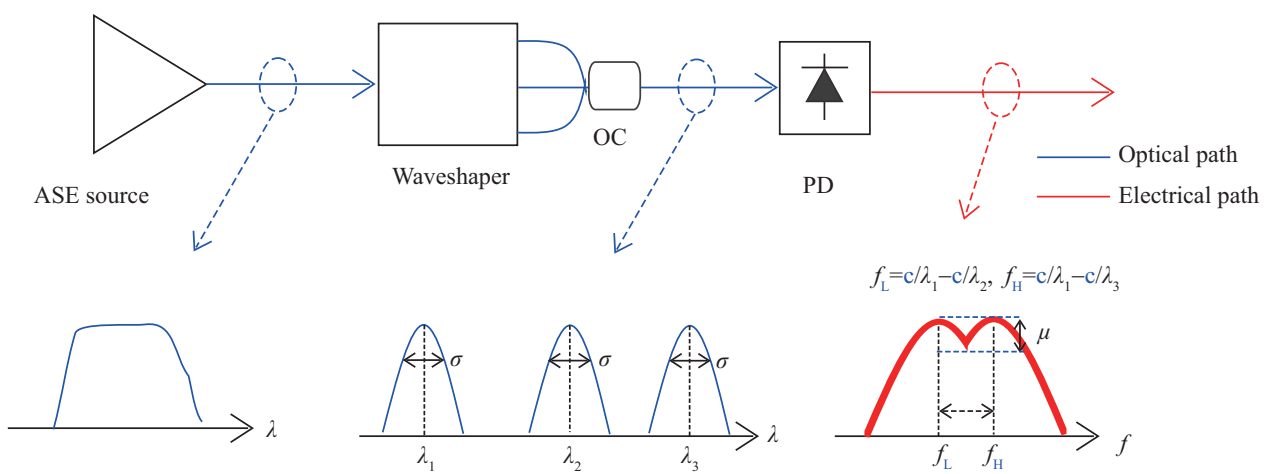

图 1 (网络版彩图) 三束不同中心波长的高斯型 ASE 光混频产生毫米波噪声信号的原理示意图

Figure 1 (Color online) Schematic diagram of millimeter-wave (mmWAVE) noise signal generation by photonic mixing of three Gaussian-shaped ASE wavelength-sliced light

根据电噪声频谱与输入噪声光谱的映射关系 ${ }^{[20,22]}$ 可知, 光电转换得到的电噪声功率谱密度 $S_{a}(f)$ 可表示为

$$
\begin{aligned}
S_{a}(f)= & \frac{2 k R_{0} \mathfrak{R}^{2}(f) P^{2}}{3 \sqrt{\pi} \sigma}\left\{\frac{1}{3} \exp \left(-\frac{\left[f-\left(v_{3}-v_{1}\right)\right]^{2}}{4 \sigma^{2}}\right)+\frac{1}{3} \exp \left(-\frac{\left[f-\left(v_{2}-v_{1}\right)\right]^{2}}{4 \sigma^{2}}\right)\right. \\
& \left.+\frac{1}{3} \exp \left(-\frac{\left[f-\left(v_{3}-v_{2}\right)\right]^{2}}{4 \sigma^{2}}\right)+\exp \left(-\frac{f^{2}}{4 \sigma^{2}}\right)\right\},
\end{aligned}
$$

其中, $k$ 为最大功率传输系数; $R_{0}$ 为系统阻抗; $\Re(f)$ 为光电探测器的响应函数; $P$ 为入射平均光功率.

式 (3) 中求和的第 3,4 项分别对应中心频率为 $v_{2}$ 与 $v_{3}$ 两束光混频产生的低频电噪声会被高频 段探测器的带通响应滤除. 因此, 由式 (3) 可推导出频率范围在 $\left[f_{\mathrm{L}}, f_{\mathrm{H}}\right]$ 内的噪声功率谱的平坦度 $\mu$, 即功率谱密度最大值与最小值之差来衡量电噪声的功率起伏, 其表达式为

$$
\begin{aligned}
\mu & =10 \log _{10}\left\{\frac{\frac{2 k R_{0} \Re^{2}(f) P^{2}}{9 \sqrt{\pi} \sigma}\left[1+\exp \left(-\frac{\left(v_{3}-v_{2}\right)^{2}}{4 \sigma^{2}}\right)\right]}{2 \cdot \frac{2 k R_{0} \Re^{2}(f) P^{2}}{9 \sqrt{\pi} \sigma} \exp \left(-\frac{\left(\frac{v_{3}-v_{2}}{2}\right)^{2}}{4 \sigma^{2}}\right)}\right\} \\
& =10 \log _{10}\left[1+\exp \left(-\frac{\left(v_{3}-v_{2}\right)^{2}}{4 \sigma^{2}}\right)\right]-10 \log _{10}\left[2 \cdot \exp \left(-\frac{\left(\frac{v_{3}-v_{2}}{2}\right)^{2}}{4 \sigma^{2}}\right)\right] .
\end{aligned}
$$

将式 (2) 代入式 (4) 可得

$$
\mu=10 \log _{10}\left[1+\exp \left(-\frac{8 \ln 2 \cdot\left(v_{3}-v_{2}\right)^{2}}{4 \Lambda^{2}}\right)\right]-10 \log _{10}\left[2 \cdot \exp \left(-\frac{2 \ln 2 \cdot\left(v_{3}-v_{2}\right)^{2}}{4 \Lambda^{2}}\right)\right] .
$$

由式 (5) 可知, 在高斯型 ASE 光的数量确定的前提下, 目标频率范围的毫米波噪声的功率谱平坦 度与滤波光谱宽度有关, 可以通过调谐滤波光谱宽度 $\Lambda$ 控制电噪声的平坦度.

为了探究 $\mathrm{D}$ 波段毫米波噪声最优平坦度, 根据市售的高速光电探测器可提供的最大工作带宽、饱 和光功率、响应度与可编程光滤波器的波长调谐分辨率 $(0.1 \mathrm{~nm})$, 数值模拟了不同滤波光谱宽度 $\Lambda$ $(0.1 \sim 0.3 \mathrm{~nm})$ 的光生毫米波噪声功率谱平坦度变化趋势. 其中, 高速光电探测器的输入光功率设定为 $10 \mathrm{dBm}$, 响应度 $\Re$ 设定为 $0.35 \mathrm{~A} / \mathrm{W}$, 高斯型光谱的中心波长设置为 $\lambda_{1}=1550 \mathrm{~nm}, \lambda_{2}=1551.04 \mathrm{~nm}$, 

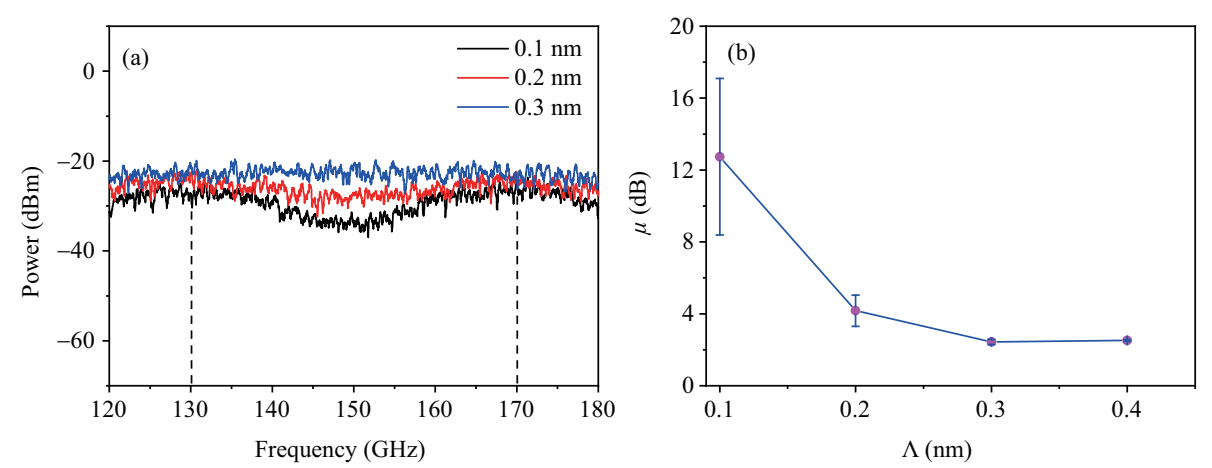

图 2 (网络版彩图) 频率范围为 $130 \sim 170 \mathrm{GHz}$ 的毫米波噪声功率谱平坦度的数值模拟结果

Figure 2 (Color online) Numerical results for the variation of the RF spectrum flatness with respect to the FWHM of optical spectra in the frequency range of $130 \sim 170 \mathrm{GHz}$. (a) Simulation of RF spectrum; (b) variation of the RF spectrum flatness with respect to the FWHM

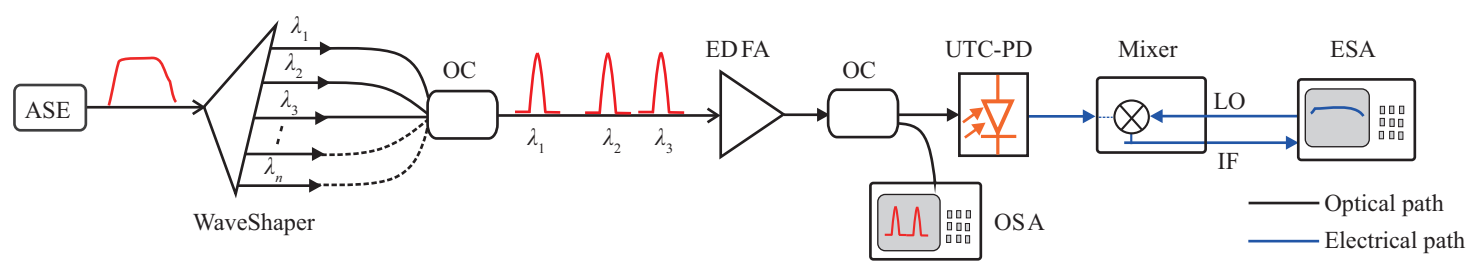

ASE: amplified spontaneous emission; WaveShaper: programmable optical processor; OC: optical coupler;

EDFA: erbium-doped fiber amplifier; OSA: optical spectrum ananlyzer; UTC-PD: uni-traveling-carrier photodiode;

Mixer: harmonic mixer; LO: local oscillator signal; IF: intermediate-frequency signal; ESA: electrical spectrum analyzer

图 3 (网络版彩图) 非相干光混频产生毫米波噪声信号的实验装置图

Figure 3 (Color online) Experimental setup for mmWave noise singal generation using multiple incoherent light mixing

$\lambda_{3}=1551.36 \mathrm{~nm}$, 根据式 (1) 与 (3), 对应电噪声 $f_{\mathrm{L}}=130 \mathrm{GHz}, f_{\mathrm{H}}=170 \mathrm{GHz}$. 如图 2(a) 所示, 随着滤 波光谱宽度 $\Lambda$ 的增加, 所产生的噪声更加平坦. 调整 $\lambda_{2}, \lambda_{3}$, 在图 2 (a) 设置基础上分别移动 $\pm 0.05 \mathrm{~nm}$, 对应 $f_{\mathrm{L}}, f_{\mathrm{H}}$ 分别改变 $6.25 \mathrm{GHz}$, 通过式 (5) 计算功率谱平坦度 $\mu$, 结果如图 2(b). 可以看出, 在 $\Lambda$ 较小 时, $\mu$ 的标准差较大, 表明该情况下的平坦度受中心波长设置影响而产生较大的起伏. $\mu$ 的平均值最小 在 $\Lambda=0.3 \mathrm{~nm}$ 处获得, 其标准差也较小, 表明功率谱平坦度最低, 受中心波长影响也小. 当 $\Lambda$ 继续增 大至 $0.4 \mathrm{~nm}$ 时, 平坦度均值有所增加, 另外, 由式 (3) 可知, 噪声功率随滤波光谱宽度增加而下降. 综 上, 基于本文提出的三光混频方法产生频率范围 130 170 GHz 的 D 波段平坦毫米波噪声过程中, 应 设置滤波光谱宽度为 $0.3 \mathrm{~nm}$, 再根据实际测量结果在 $\pm 0.05 \mathrm{~nm}$ 范围内适当调整中心波长 $\lambda_{2}, \lambda_{3}$.

\section{3 实验装置与结果}

\section{1 实验装置}

实验装置如图 3 所示, 其中使用可编程光滤波器 (Finisar, Waveshaper 4000A) 对线宽为 $40 \mathrm{~nm}$ 的 ASE 光源整形滤波, 输出三束通道滤波光谱宽度相同、中心波长不同的高斯型 ASE 光. 再将所 有通道的非相干光耦合, 为了提高噪声功率, 通过掺铒光纤放大器 (EDFA) 对耦合后的光功率放大至 $10 \mathrm{dBm}$. 最后在 UTC-PD (NTT, IOD-PMD-14001, 130 170 GHz, $\Re=0.35 \mathrm{~A} / \mathrm{W}$ ) 上进行光电转换, 产 生 130 170 GHz 平坦的 D 波段毫米波噪声信号. 

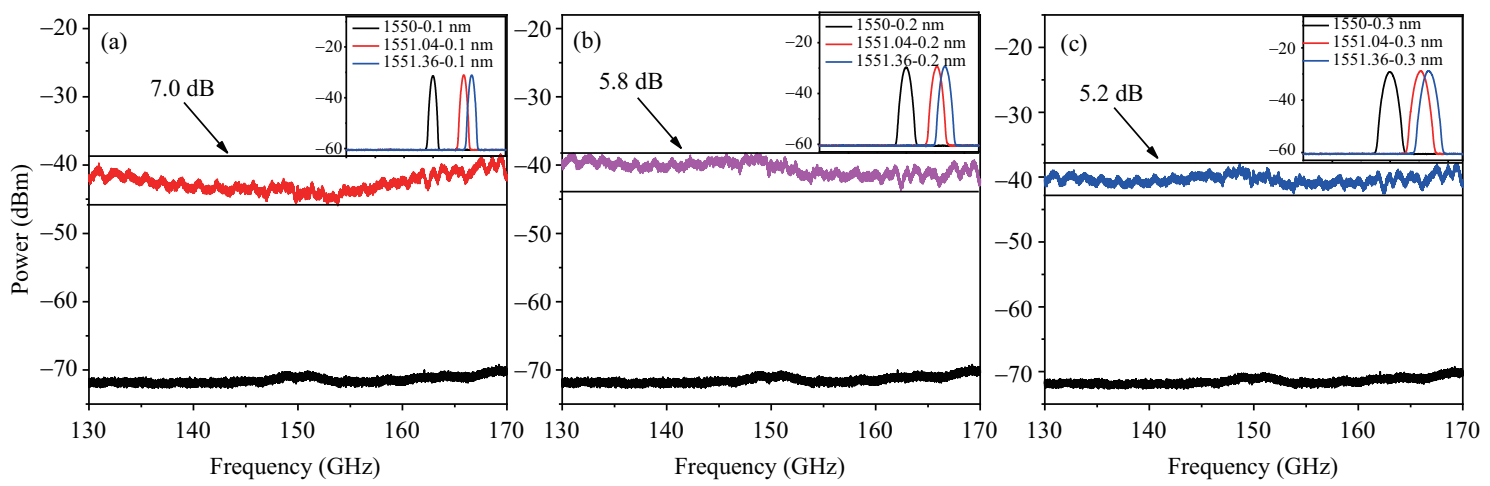

图 4 (网络版彩图) 不同滤波光谱宽度的三光混频产生频率范围 130 170 GHz 的噪声功率谱

Figure 4 (Color online) RF spectrum of mmWave noise in the frequency range of 130 170 GHz using three Gaussianshaped ASE wavelength-sliced light mixing with different FWHM. (a) FWHM $=0.1 \mathrm{~nm}$; (b) FWHM $=0.2 \mathrm{~nm}$; (c) FWHM $=0.3 \mathrm{~nm}$

实验所产生的 D 波段毫米波噪声信号通过 UTC-PD 的输出波导 (WR-6.0) 耦合进混频器 (Mixer, VDI-731, 110-170 GHz) 的输入波导 (WR-6.5) 进行下变频转换, 再利用频谱分析仪的中频 (intermediatefrequency, IF) 输入端对下变频转换后的毫米波噪声信号实时测量.

\section{2 实验结果}

图 4 所示实验结果为不同滤波光谱宽度的三光混频得到的噪声功率谱. 可编程光滤波器控制三路 高斯型 ASE 光的中心波长: $\lambda_{1}=1550 \mathrm{~nm}, \lambda_{2}=1551.04 \mathrm{~nm}, \lambda_{3}=1551.36 \mathrm{~nm}$, 以 $0.1,0.2,0.3 \mathrm{~nm}$ 的 滤波光谱宽度进行 3 组实验. 对比图 4(a) (c) 可得, 不同滤波光谱宽度的光混频都可产生频率范围 130 170 GHz 的毫米波噪声信号, 而且与理论分析结果趋势一致, 滤波光谱宽度增加, 噪声平坦度降 低. 实验结果显示, 滤波光谱宽度为 $0.3 \mathrm{~nm}$ 时得到的噪声功率谱平坦度约为 $5.2 \mathrm{~dB}$.

根据理论推导与数值模拟分析, 在实验中保持滤波光谱宽度为 $0.3 \mathrm{~nm}$ 的前提下, 对 $\lambda_{2}, \lambda_{3}$ 的设 置在图 4 所示的基础上进行 $\pm 0.05 \mathrm{~nm}$ 的微调, 进一步优化噪声功率谱的平坦度. 对比图 5(a) (c), 尽 管滤波光谱宽度保持不变, 中心波长 $\lambda_{2}, \lambda_{3}$ 的微调, 会使得噪声功率谱的平坦度发生改变, 与理论计 算的噪声功率谱最低所对应的中心波长数值有一定偏离, 主要原因在于, UTC-PD 的频率响应曲线并 非均匀一致 ${ }^{[23]}$. 除此之外, 测量设备, 如频谱仪本身内部的噪声, 会随着频率升高而增强, 混频器自身 的损耗曲线会导致信号相应衰减, 这两个因素也会对高频毫米波噪声的功率谱测量结果产生影响. 如 图 5(c) 所示, 根据中心波长的调整, 产生的 130 170 GHz 的 D 波段毫米波噪声功率谱平坦度最低约 为 $3.9 \mathrm{~dB}$.

超噪比 (ENR) 是噪声源的一个重要指标, 它与噪声功率谱密度的换算关系可表示为

$$
\mathrm{ENR}=10 \log _{10}\left(\frac{T_{h}-T_{\mathrm{c}}}{T_{0}}\right)=S_{a}(f)-S_{0}(f),
$$

其中, $T_{\mathrm{h}}, T_{\mathrm{c}}$ 分别为噪声源工作与关闭状态下的等效输出噪声温度, $T_{0}$ 为标准室温 $(290 \mathrm{~K}), S_{a}(f)$, $S_{0}(f)$ 分别为噪声源工作与关闭状态下的功率谱密度.

图 5(c) 所对应的超噪比如图 6 所示, 在中心波长分别为 $\lambda_{1}=1550 \mathrm{~nm}, \lambda_{2}=1551.06 \mathrm{~nm}, \lambda_{3}=$ $1551.34 \mathrm{~nm}$, 滤波光谱宽度 $\Lambda=0.3 \mathrm{~nm}$ 的条件下, 通过三光耦合混频在 $130 \sim 170 \mathrm{GHz}$ 产生了超噪比 $>35 \mathrm{~dB}$, 平坦度低至 $\pm 2.5 \mathrm{~dB}$ 的毫米波噪声信号. 据我们所知, 这是毫米波段噪声发生器迄今为止所 

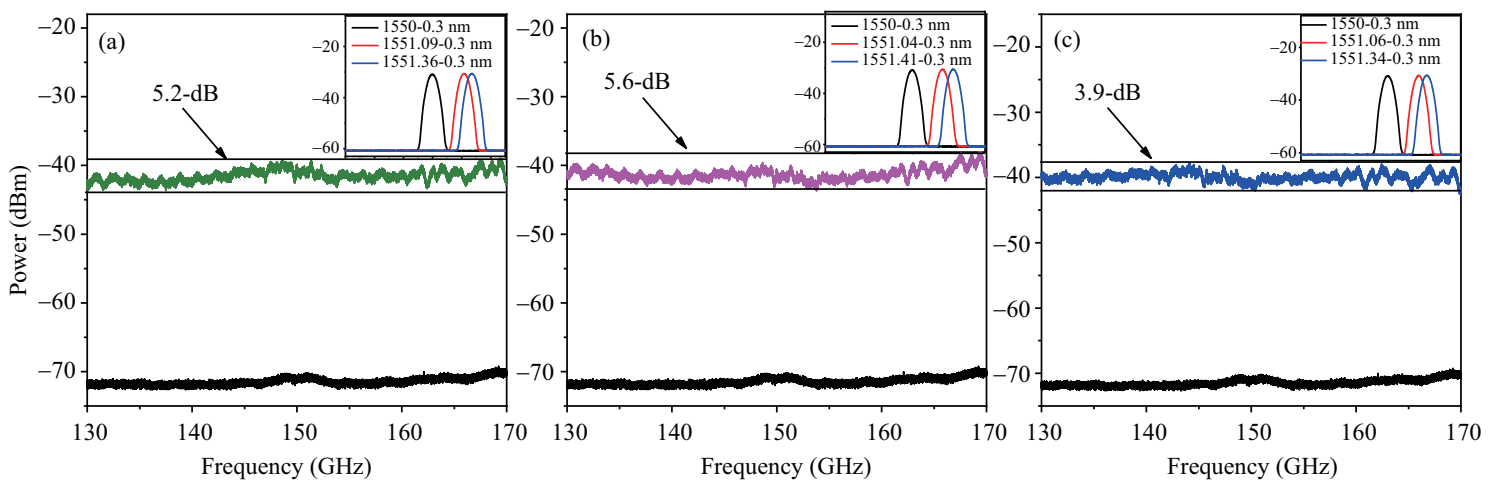

图 5 (网络版彩图) 基于中心波长 $\lambda_{2}, \lambda_{3}$ 微调的噪声功率谱 (滤波光谱宽度 $\Lambda=0.3 \mathrm{~nm}$ )

Figure 5 (Color online) RF spectrum of mmWave noise in the frequency range of 130 170 GHz with fine-tune $\lambda_{2}, \lambda_{3}$ (FWHM $\Lambda=0.3 \mathrm{~nm}$ ). (a) Central wavelength are 1550, 1551.09, and $1551.36 \mathrm{~nm}$; (b) central wavelength are 1550, 1551.04, and $1551.41 \mathrm{~nm}$; (c) central wavelength are 1550, 1551.06, $1551.34 \mathrm{~nm}$.

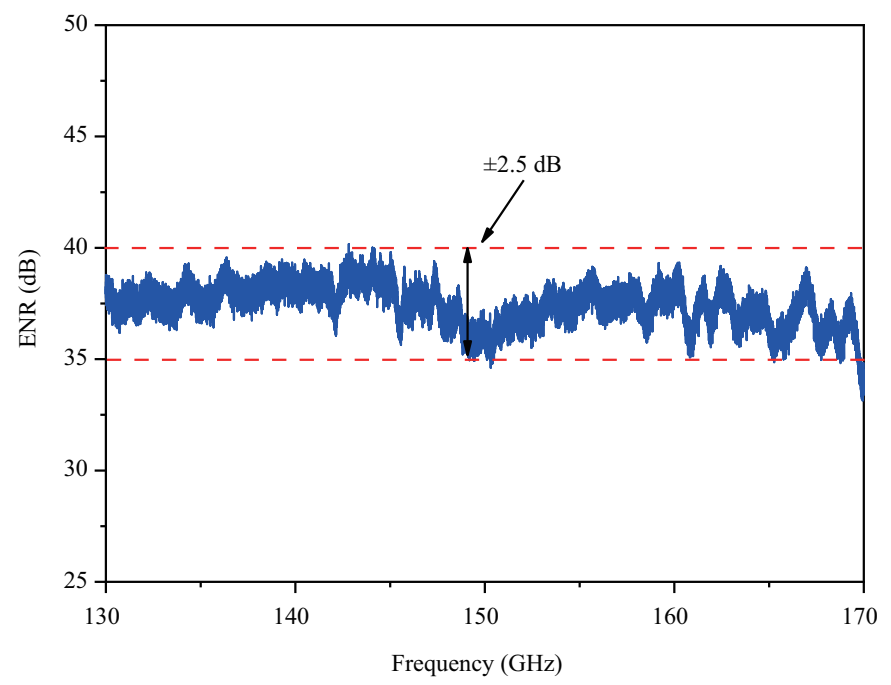

图 6 (网络版彩图) 频率范围 $130 \sim 170 \mathrm{GHz}$ 的毫米波噪声超噪比实验结果

Figure 6 (Color online) ENR of mmWave noise in the frequency range of 130 170 GHz

取得的最平坦的超噪比指标. 本文方法所产生的连续平坦毫米波噪声对 D 波段接收机的噪声系数测 试及设备动态范围校准具有优势.

\section{4 结论}

本文提出了一种基于光子混频技术产生 $\mathrm{D}$ 波段平坦毫米波噪声信号的新方法, 并通过实验验证 了通过调控用于混频的三束高斯型 ASE 光的中心波长与滤波光谱宽度, 优化毫米波噪声的平坦度, 实 现了 D 波段范围内 $(130 \sim 170 \mathrm{GHz})$ 超噪比 $>35 \mathrm{~dB}$ 、平坦度低至 $\pm 2.5 \mathrm{~dB}$ 的毫米波噪声信号的稳 定输出. 利用响应频段更高的光电探测器, 基于本文方法能实现更高频率 (太赫兹波段)、更大带宽范 围 (上百 $\mathrm{GHz}$ 以上) 内的平坦毫米波噪声信号的产生. 此外, 本文工作首次展示了 ASE 非相干光源 与 UTC-PD 相结合混频产生高频连续平坦毫米波噪声信号的实验结果, 有望为未来高频毫米波器件 
的研究及性能评估提供技术与硬件支撑.

\section{参考文献}

1 Ando I, Tanio M, Ito M, et al. Wireless D-band communication up to 60 Gbit/s with 64QAM using GaAs HEMT technology. In: Proceedings of IEEE Radio and Wireless Symposium (RWS), Austin, 2016. 193-195

2 Mittleman D M. Twenty years of terahertz imaging. Opt Express, 2018, 26: 9417-9431

3 Jaeschke T, Bredendiek C, Kuppers S, et al. High-precision D-band FMCW-radar sensor based on a wideband SiGetransceiver MMIC. IEEE Trans Microw Theor Techn, 2014, 62: 3582-3597

4 Liu J, Jiang J, Cheng B B, et al. D-band transmittance of typical clothing. Acta Opt Sin, 2016, 36: 100-104 [刘杰, 蒋均, 成涁涁, 等. 典型衣物的 D 波段透射率研究. 光学学报, 2016, 36: 100-104]

5 Rappaport T S, Xing Y C, Kanhere O, et al. Wireless communications and applications above $100 \mathrm{GHz}$ : opportunities and challenges for 6G and beyond. IEEE Access, 2019, 7: 78729-78757

6 Yang S Y, Yu W H, An S N, et al. A D-band communication transmitter module with a novel self-aligned microstrip line-to-waveguide transition. J Infrared Millim Wave, 2019, 38: 296-302 [杨宋源, 于伟华, 安思宁, 等. 一种基于新型 自组装微带 - 波导过渡的 D 波段通信发射机模块. 红外与毫米波学报, 2019, 38: 296-302]

7 Shahriar C, Pan M L, Lichtman M, et al. PHY-layer resiliency in OFDM communications: a tutorial. IEEE Commun Surv Tut, 2015, 17: 292-314

8 Eichen E, Schlafer J, Rideout W, et al. Wide-bandwidth receiver/photodetector frequency response measurements using amplified spontaneous emission from a semiconductor optical amplifier. J Lightw Technol, 1990, 8: 912-916

9 Pepe D, Barnett C, D'Amore G, et al. On-chip millimeter-wave cold-source noise figure measurements with PNA-X. IEEE Trans Instrum Meas, 2017, 66: 3399-3401

10 Liu J Q, Han S L, Meng X, et al. Radiometric calibration method of 2 14 $\mu$ m infrared spectroradiometer. Acta Opt Sin, 2019, 39: 168-174 [刘加庆, 韩顺利, 孟釒金, 等. 一种 2 14 $\mu \mathrm{m}$ 红外光谱辐射计的辐射定标方法. 光学学报, 2019, 39: $168-174]$

11 Paik H, Sastry D N N, SantiPrabha D I. Effectiveness of noise jamming with white Gaussian noise and phase noise in amplitude comparison monopulse radar receivers. In: Proceedings of IEEE International Conference on Electronics, Computing and Communication Technologies (CONECCT), 2014. 1-5

12 Ehsan N, Piepmeier J, Solly M, et al. A robust waveguide millimeter-wave noise source. In: Proceedings of the 45th European Microwave Conference (Eumc), 2015. 853-856

13 Goncalves J C A, Ghanem H, Bouvot S, et al. Millimeter-wave noise source development on SiGe BiCMOS 55-nm technology for applications up to $260 \mathrm{GHz}$. IEEE Trans Microw Theor Techn, 2019, 67: 3732-3742

14 Diebold S, Weissbrodt E, Massler H, et al. A W-band monolithic integrated active hot and cold noise source. IEEE Trans Microw Theor Techn, 2014, 62: 623-630

15 Kantanen M, Weissbrodt E, Varis J, et al. Active cold load MMICs for Ka-, V-, and W-bands. IET Microw Antenn Propagat, 2015, 9: 742-747

16 Coen C T, Frounchi M, Lourenco N E, et al. A 60-GHz SiGe radiometer calibration switch utilizing a coupled avalanche noise source. IEEE Microw Wirel Compon Lett, 2020, 30: 417-420

17 Duan G H, Gorgiev E. Non-white photodetection noise at the output of an optical amplifier: theory and experiment. IEEE J Quantum Electron, 2001, 37: 1008-1014

18 Nagatsuma T, Kumashiro T, Fujimoto Y, et al. Millimeter-wave imaging using photonics-based noise source. In: Proceedings of the 34th International Conference on Infrared, Millimeter, and Terahertz Waves, Busan, 2009. $1-2$

19 Vidal B. Broadband photonic microwave noise sources. IEEE Photon Technol Lett, 2020, 32: 592-594

20 Song H J, Shimizu N, Kukutsu N, et al. Microwave photonic noise source from microwave to sub-terahertz wave bands and its applications to noise characterization. IEEE Trans Microw Theor Techn, 2008, 56: 2989-2997

21 Sun Y H, Chen Y X, Li P, et al. Flat millimeter-wave noise generation by optically mixing multiple wavelength-sliced ASE lights. IEEE Photon Technol Lett, 2021, 33: 1270-1273

22 Nazarathy M, Sorin W V, Baney D M, et al. Spectral analysis of optical mixing measurements. J Lightw Technol, 1989, 7: 1083-1096 
23 Furuta T, Ito T, Muramoto Y, et al. D-band rectangular-waveguide-output uni-travelling-carrier photodiode module. Electron Lett, 2005, 41: 715-716

\title{
Generation of $130-170 \mathrm{GHz}$ flat millimeter-wave noise signal
}

Yuehui SUN ${ }^{1}$, Ya GUO ${ }^{2}$, Yuncai WANG ${ }^{1 *}$, Wenjie LIU $^{1}$, Haibi HUANG ${ }^{1}$, Yimin HUANG ${ }^{1}$ \& Yuwen QIN $^{1}$

1. School of Information Engineering, Guangdong Provincial Key Laboratory of Photonics Information Technology, Guangdong University of Technology, Guangzhou 510006, China;

2. School of Electronics and Information, Northwestern Polytechnical University, Xi'an 710072, China

* Corresponding author. E-mail: wangyc@gdut.edu.cn

\begin{abstract}
Three incoherent light-mixing method of the D-band flat millimeter-wave (mmWave) noise signal generation is proposed. The frequency ranges and RF spectrum flatness are effectively controlled concurrently by the center wavelengths and the 3 - $\mathrm{dB}$ bandwidths of three light beams for the photonic mmWave noise signal generation using a high-speed photodetector. The relationship between the RF spectrum flatness, central wavelength difference, and 3-dB bandwidth of Gaussian-type incoherent light is theoretically analyzed. The experimental results show that the D-band $(130-170 \mathrm{GHz})$ mmWAVE noise signal with excess noise ratio $>35 \mathrm{~dB}$ and flatness as low as $\pm 2.5 \mathrm{~dB}$ is generated with a uni-traveling-carrier photodetector as the photomixer.
\end{abstract}

Keywords noise generator, millimeter-wave noise, photonic mixing, excess noise ratio, flatness 